\title{
Designing a Bidirectional Workflow for OpenStreetMap Data Integration in an INSPIRE Based SDI
}

\author{
Silva, António José \\ ISEGI - Universidade Nova de Lisboa \\ Universidade do Minho \\ 4710-057 Braga, Portugal \\ (+351) 253604470 \\ d2011090@isegi.unl.pt
}

\author{
Rocha, Jorge Gustavo \\ Departamento de Informática \\ Universidade do Minho \\ 4710-057 Braga, Portugal \\ (+351) 253604470 \\ jgr@di.uminho.pt
}

\begin{abstract}
More educated people, with more sophisticated devices, are creating new trends: crowded source data and citizen science. Citizens are able to capture data and to reason about it. They are producers and consumers of their own data. Meanwhile, local administration is struggling with less resources and more pressure to become more efficient. In this paper we try to bridge the gap between these two different worlds, apart from each other, proposing a workflow. We discuss the advantages and the technical challenges we might face if crowded source data can be used by the administration. To make this possible, we take advantage of the existing European regulation, the INSPIRE directive, and designed a workflow to implement the rules regarding data specifications to the OpenStreetMap (OSM) data to combine it seamless with official existing datasets.
\end{abstract}

\section{Categories and Subject Descriptors}

H.2.8 [Information Systems]: Database management - database applications; K.4.1 [Computer Milieux]: Computers and Society - public policy issues

\section{General Terms}

Human Factors, Standardization

\section{Keywords}

INSPIRE; Geospatial Crowdsourcing; SDI; OpenStreetMap

\section{INTRODUCTION}

Nowadays, all over the world, local authorities are required to do more with fewer resources. On the other hand, citizens have higher expectations on services and with its quality, but no desire to see increases in taxes. With great responsibilities in a wide range of domains, local authorities need to make the most of their information to deal with these challenges. One common key theme through nearly all these domains is 'where' they happen.

The address is the most important and simple key to solve this 'where' problem. An address is a collection of information, presented in a mostly fixed format, used for describing the location of a building, structure, or a plot of land, generally using political boundaries and street names as references.

Every local and national authority should hold local address

Permission to make digital or hard copies of all or part of this work for personal or classroom use is granted without fee provided that copies are not made or distributed for profit or commercial advantage and that copies bear this notice and the full citation on the first page. Copyrights for components of this work owned by others than ACM must be honored. Abstracting with credit is permitted. To copy otherwise, or republish, to post on servers or to redistribute to lists, requires prior specific permission and/or a fee. Request permissions from Permissions@ acm.org.

ICEGOV2014, October 27 - 30, 2014, Guimaraes, Portugal

Copyright 2014 ACM 978-1-60558-611-3/14/10...\$15.00

http://dx.doi.org/10.1145/2691195.2691308 information and also should be capable of exploiting its potential to save money and deliver better services to citizens and companies. With different organic units and departments delivering different services to the same address it makes sense to ensure that everyone is sharing the same location intelligence. Thus, it becomes clear that an open centralized repository of addresses, would greatly improve in solving this and other problems related to this topic with benefits to citizens, local and central administration and companies.

With the most recent technological advances and lower prices in GPS units, cameras, smart phones, sensor networks, RFID (radio frequency identification) readers, higher-capacity domestic Internet connections, and computers, especially all of the technologies freely called Web 2.0, a new communication paradigm were introduced [14], where users can also be information providers. These events made geospatial technologies mobile and ubiquitous and have completely transformed how geographic data are collected, stored, disseminated, analyzed, visualized, and used, especially as government mapping and updating declines $[7,9,15,16]$.

Users and therefore companies were the first to understand this phenomenon, geospatial crowdsourcing. Many governments are considering how they can better engage with and accept citizen input online, particularly through the gathering and use of geospatial crowdsourcing which is considered a very beneficial process to decision-making support [4, 13] because the data collected by users can help correct errors, fill in gaps [9] or even to know and predict people's activities [10]. However, such efforts are still in their early stages [3].

Using this kind of data represents a shift of paradigm on how spatial data are created, moving from an authoritative process of geographic data production involving experts to informal processes that engage large numbers of amateurs with varying interests and abilities [4].

As expected, geospatial crowdsourcing data is growing at an incredible pace, faster than the development of workflows able to take full advantage of it[1], pointing the idea that this kind of data can be part of future real-time Spatial Data Infrastructures (SDIs) and serve as an input for spatial decision-making processes.

In this context, a lot of effort has been invested, around the world, in (SDIs) during the last decade regarding interoperable standards for services and data [2]. Different SDI architectures have caused new obstacles. To integrate SDIs internationally, in Europe a major development has been the entering in force of the INSPIRE Directive, establishing an SDI in Europe.

Interoperability is becoming essential for the today's geographic information systems (GIS). Geographic information (GI) is 
usually available as datasets stored in databases and accessible via GIS. However, and largely due to geospatial crowdsourcing, these information sources are increasingly heterogeneous and show differences in data formats, database schema and object concepts. The implementation of SDIs such as INSPIRE enables accessibility and the sharing of geographic data and interoperability among the systems [8].

This represents a great opportunity to integrate geospatial crowdsourcing, OSM data in this case, in the SDIs workflow in order to turn geospatial crowdsourcing in a valuable source of information.

With this work, we want to update the concept of INSPIRE SDI, in order to include a new workflow, capable to take full advantage from Crowdsourced data, in particular taking as its starting point the case study of postal addresses. We want to develop an INSPIRE based Open Service of Postal addresses integrating free and available data from OSM with official data sources. In order to achieve this, we need to overcome several interesting challenges.

We believe that the integration of geospatial data crowdsourcing in general, and the OSM data on INSPIRE based SDIs will be a trend in the short term and more cases of this integration will emerge.

\section{STATE OF THE ART 2.1 Geospatial Crowdsourcing}

With this rapid growth of broader interest and engagement, geographic information collected by common people has been studied from several viewpoints, which led to the emergence of different concepts to describe this new trend, ranging from Geospatial Crowdsourcing to User-generated content, from Geoweb to Volunteered Geographic Information (VGI), from Neogeography to Citizen Science or ubiquitous cartography, however the main idea resides around the usage of the Internet to create, share, and analyze geographic information $[1,6,7,14]$.

\subsection{INSPIRE}

INSPIRE, the acronym for the INfrastructure for SPatial InfoRmation in Europe is a European Commission directive that primary aims at establishing a European infrastructure of geographic data to support environmental policy. This directive addresses 34 spatial data themes, with key components specified through technical implementing rules [11]. INSPIRE is an answer in terms of meeting the need for the sharing, access and exchange of geographic data among member states by implementing a common data specification [8]. The major goal is to enable the sharing of environmental information among public sector bodies and better enable public access to spatial data across Europe by means of an on-line service offering data and metadata. With all this being said it is believed that various stakeholders will benefit with INSPIRE in the future.

\subsubsection{INSPIRE Addresses Data Specifications}

Nowadays, an address is probably the most common and used way by people to describe a location. Geographically speaking, an address can be seen as a collection of information, presented in a mostly fixed format, used for describing the location of a building, generally using political boundaries and street names as references, along with other identifiers such as house or apartment numbers.

According to the INSPIRE Address data specification document, this is required to facilitate the interoperability of address information between the member states. Although all national or local address systems share similar concepts and general properties, differences exist in formal and informal standards, rules, schemes and data models within Europe [13].

The overall concept of this data specification is that an address has a "locator", e.g. an address number that enables a user to distinguish it from the neighbor addresses; and a geographic position, which enables an application to locate the address spatially.

To identify the address unambiguously in a wider context an address must be associated with a number of "address components" that define its location within a certain geographic area. Each of the address components represents a spatial identifier as for example the name of a road, district, postcode, municipality, region or country.

\section{CHALLENGES}

As stated previously, the main goal of this work is the development of a workflow that allows integrating data from OSM and official sources in an INSPIRE based SDI. In order to achieve it, is of extreme importance to have a clear awareness of the challenges we have to overcome. These challenges can be of several types.

\subsection{Data Models}

Typically, the term data model is generally used to describe the objects represented by a computer system together with their properties and relationships.

There are important differences between the OSM data model and the purposed INSPIRE data model, that are important to understand, in order to be able of better design a workflow capable to transform OSM data into INSPIRE and its opposite if possible.

\subsubsection{OSM Data Model}

The OSM data model it is quite simple, because of its nature is very dynamic, interactive, effective and flexible. In OSM data model, geometries are represented by nodes, describing georeferenced points. Several nodes can be further combined to create so called ways, describing either lines or polygons. More complex polygons, such as those with holes, or complex relationships between OSM features, such as turn restrictions or tours, can be expressed by using relations.

Although not their most visible characteristic but probably one of the most important, OSM does not only contain information about natural areas or streets but also contain various kinds of semantic information, such as addresses and building types, such as the building height among many others. For the attributes representation of an OSM feature, it is possible to tag the geometry with key-value pairs. Thereby, the key describes some kind of information domain or characteristic (e.g., highway, building, etc.) and the value refines the information (e.g., service road, university, etc.). Due to its open and constantly evolving character, the amount of key-value pairs is unlimited as well as the key-value pairs themselves [5].

Unfortunately, despite the OSM data model is best suited for visualization and data editing, it is quite problematic to use it for data analysis because it's quite redundant, resultant by the openness of the model to contributors, which causes frequent changes and misconceptions in the correct cataloging data. 


\subsubsection{INSPIRE Data Model}

Due to its nature, a politically driven top-down approach, with strict rules to support it, the opposite of OSM, the INSPIRE data model it's more suited to represent a more hierarchical model. The INSPIRE directive have its data model very well defined, specified and documented for each spatial data themes.

\subsection{Data Inconsistencies}

The inconsistency of the data is a recognized problem in databases, widely studied and it is generally difficult to solve, it is extremely important to minimize, to ensure as much as possible integrity and consequently the quality of the data.

\subsubsection{OSM Data Inconsistencies}

When the OSM data model was presented, it was clear, that because of its collaborative nature and continuous updating, this model it's more prone to the appearance of errors and inconsistencies. Specifically these inconsistencies can arise from different practices, sources and limitations of the model itself. The most common are: Change of ID's; Broken relations (foreign keys); Generated by poor tagging.

\subsection{Semantics}

The growth of available online geographic information raises the issue of semantics. In the OSM vector data set, map objects are associated with properties that encode their semantic content. In OSM, the properties of an object are called 'tags'. The meaning and usage of these tags are negotiated within the contributors' community on the OSMWiki website. The meaning of the tags can change over time, in a process of emergent semantics, where concepts emerge, shift and disappear in a complex evolutionary negotiation [1].

Different terms may refer to similar concepts, while the same terms may refer to different concepts. This causes semantic heterogeneity in the dataset. In this context, the most promising approach would be the development and the implementation of geospatial semantics by means of ontology in the geospatial dataset to overcome this kind of ambiguity [8].

\subsection{Data Comparison}

Because of the differences pointed above, it is very difficult to compare datasets from OSM with official data sources from the same area. In addition, geographical and topological differences, increases the difficulty of perception.

\subsection{Bidirectionality}

Until now it was only discussed the data flow in one direction from OSM to INSPIRE. Another important challenge that we want to study and overcome it's the bidirectionality. In this context, this concept refers to the ability of total interoperability between OSM and INSPIRE, ie, the transformation of data will not occur only in the direction from OSM to the INSPIRE but also its reverse. This means that, to be able to take full advantage of OSM in this process, it is also necessary to ensure the existence of flows from INSPIRE to OSM in order to add value for users and for the OSM data, without jeopardizing the spirit of the OSM project and especially on their community.

\section{ARCHITECTURE}

The main goal of this work it is the design a workflow to insert geospatial crowdsourcing data OSM in INSPIRE. The workflow shown on the image below, evidences the official and the authoritative nature of INSPIRE with its rules, represented by the annexes, feeding the database and its metadata. From this structure web services are developed, these are used in applications and geoportals, and are thus presented to the citizen. On the other hand, the citizen is the central piece of the Geospatial Crowdsourcing, because they are responsible for creating the OSM data and simultaneously consumers of such data. It is interesting to note the active role of the citizen with the OSM (producer and user - "produser"), as opposed to the passive role within INSPIRE (only consumer).

The OSM database is then processed with ETL (Extract, Transform and Load) processes to transform it into an intermediate database and metadata repository INSPIRE based. From this database, is then possible to integrate these data into INSPIRE SDI, thus continuing the cycle described above.

With this workflow it is possible to integrate data from geospatial crowdsourcing in INSPIRE, as the citizen gets a new role, a bidirectional role, even if only indirectly, in relation to INSPIRE SDI based.

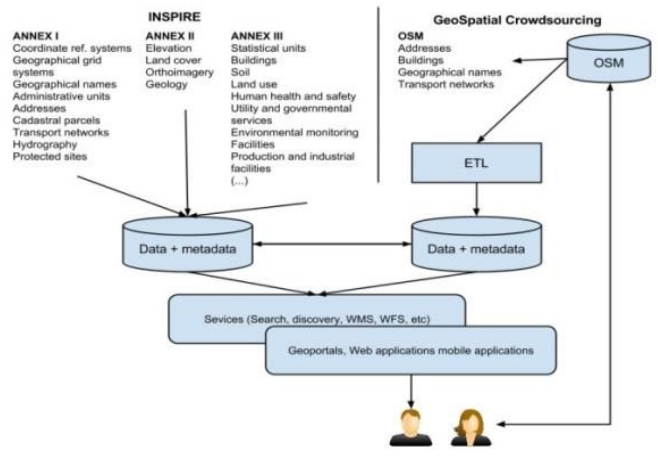

Figure 1 - Proposed workflow

\section{CASE STUDY}

In the context of this work, we tested this workflow to transform OSM and official data into INSPIRE addresses scheme data model. For the initial test, we used the official data provided by one municipality, Águeda, and the data from the same area from OSM. This test was carried out just to see the addresses from OSM and from the municipality side by side. It was also used to measure how hard is the transformation process is.

To transform the OSM representation to INSPIRE, more datasets were necessary. The INSPIRE model creates many relations among the datasets, which is a useful and powerful mechanism to ensure data consistency. Addresses are related to Administrative Units (see Fig. 1), for example. But to relate addresses with Administrative Units we need official IDs to link to the related Administrative Unit, which are not available in Portugal. In fact, there are no official INSPIRE datasets in Portugal. For this case study, before starting processing addresses, we created an INSPIRE based datasets with the Administrative Units and from the Postal Codes. For each administrative unit and postal code, unique identifiers were created. Each identifier is unique for a known time period, since administrative units and postal codes change over time. These related datasets were inserted in a spatial relational database management system (SRDBMS) so as to be possible to perform proper processing on these datasets.

Various processing tasks were performed in order to prepare the OSM data for subsequent procedures. These are composed of a series of ETL processes which generically transform the OSM data to the address feature type in Geography Markup Language (GML) format, according to the INSPIRE data model. 
With these transformations, a single address, usually written in three or four lines (street name, building and postal code) of text, is now described in more than 200 of GML lines, which demonstrates the complexity of the INSPIRE data model.

Official addresses provided by the municipality were also transformed to the INSPIRE data model.

After these transformations we were able to put OSM and official addresses side by side. It is not easy to compare both datasets, as we already anticipated in our discussion about difficulties and challenges. The lineage of the datasets is so different, that more powerful tools must be used to support a continuous workflow where crowded source data can be exploited by the local or central administration.

\section{CONCLUSIONS}

With this work we think have proven the interest of a workflow to integrate geospatial crowdsourcing data (OSM) with INSPIRE, using this directive as the common denominator and an alternative to confront official data. With this workflow it is possible to better understand the new role of users in the INSPIRE based SDI's, making INSPIRE data better accessible for citizens. INSPIRE was created before this recent increase of crowded data and citizen science.

The best of OSM is the database. Integrating OSM in INSPIRE it's possible to take the best of the greater value from OSM, the data.

The INSPIRE data model is much more powerful, but adds more complexity. In the medium term is necessary to develop new tools to help us manipulate these INSPIRE based data sets, namely at the level GIS software, without seeing the underlying complexity.

This is an ongoing work, so in order to better overcome the challenges identified in this work, it will be necessary to perform some adjustments to the workflow.

In addition to the addresses, there are more INSPIRE schemes that should be derived from OSM dataset, such as buildings, geographical names and transport networks and the INSPIRE components that uses these themes must or should be redesigned.

Despite the local nature of this work, the effort made in this work can and should be replicated throughout Europe. This is not a closed process and there are many tests and tasks to be performed in order to fully implement this workflow. Hopefully, if this work is replicated in other places, the workflow can be further improved and became more flexible and robust.

It will be important, in the near future, to see how INSPIRE reflects local, regional and national needs. Currently, there is low awareness on regional level and the benefits for the local level are not clearly defined.

\section{REFERENCES}

[1] Ballatore, A. et al. 2012. Geographic knowledge extraction and semantic similarity in OpenStreetMap. Knowledge and Information Systems. 37, 1 (Oct. 2012), 61-81.

[2] Baranski, B. et al. 2011. Matching INSPIRE Quality of Service Requirements with Hybrid Clouds. Transactions in GIS. 15, (Jul. 2011), 125-142.

[3] Coleman, D.J. 2013. Potential Contributions and Challenges of VGI for Conventional Topographic Base-Mapping Programs. Crowdsourcing Geographic Knowledge:
Volunteered Geographic Information (VGI) in Theory and Practice. 245-263.

[4] Feick, R. and Roche, S. 2013. Understanding the Value of VGI. Crowdsourcing Geographic Knowledge: Volunteered Geographic Information (VGI) in Theory and Practice. D. Sui et al., eds. Springer Netherlands. 15-29.

[5] Goetz, M. 2013. Towards generating highly detailed 3D CityGML models from OpenStreetMap. International Journal of Geographical Information Science. 27, 5 (May 2013), 845-865.

[6] Goetz, M. and Zipf, A. 2013. The Evolution of GeoCrowdsourcing: Bringing Volunteered Geographic Information to the Third Dimension. Crowdsourcing Geographic Knowledge: Volunteered Geographic Information (VGI) in Theory and Practice. D. Sui et al., eds. Springer Netherlands.

[7] Goodchild, M.F. 2007. Citizens as sensors: the world of volunteered geography. GeoJournal. 69, 4 (Nov. 2007), 211221.

[8] Gunay, A. et al. 2013. Building a semantic based public transportation geoportal compliant with the INSPIRE transport network data theme. Earth Science Informatics. 7, 1 (Aug. 2013), 25-37.

[9] Haklay, M. 2013. Citizen Science and Volunteered Geographic Information: Overview and Typology of Participation. Crowdsourcing Geographic Knowledge: Volunteered Geographic Information (VGI) in Theory and Practice. D. Sui et al., eds. Springer Netherlands. 105-122.

[10] Harvey, F. 2013. To Volunteer or to Contribute Locational Information? Towards Truth in Labeling for Crowdsourced Geographic Information. Crowdsourcing Geographic Knowledge: Volunteered Geographic Information (VGI) in Theory and Practice. D. Sui et al., eds. Springer Netherlands. $31-42$.

[11] Infrastructure for Spatial Information in the European Community: 2014.

http://inspire.ec.europa.eu/index.cfm/pageid/48. Accessed: 2014-05-21.

[12] INSPIRE - Data Specifications for Addresses: 2009.

[13] Johnson, P.A. and Sieber, R.E. 2013. Situating the Adoption of VGI by Government. Crowdsourcing Geographic Knowledge: Volunteered Geographic Information (VGI) in Theory and Practice. D. Sui et al., eds. Springer Netherlands. 65-81.

[14] Sui, D. et al. 2013. Volunteered Geographic Information , the Exaflood, and the Growing Digital Divide. Crowdsourcing Geographic Knowledge: Volunteered Geographic Information (VGI) in Theory and Practice. 1-12.

[15] Thatcher, J. 2013. From Volunteered Geographic Information to Volunteered Geographic Services. Crowdsourcing Geographic Knowledge: Volunteered Geographic Information (VGI) in Theory and Practice. D. Sui et al., eds. Springer Netherlands. 161-173.

[16] Zielstra, D. and Zipf, A. 2010. A comparative study of proprietary geodata and volunteered geographic information for germany. 13th AGILE International Conference on Geographic Information Science (2010). 